\title{
Dynamics of a Nonlinear Business Cycle Model Under Poisson White Noise Excitation
}

\author{
Jiaorui LI \\ Xi'an Statistical Research Institute, Xi'an University of Finance \& Economics, Xi'an 710100, China \\ E-mail: Jiaoruili@xaufe.edu.cn \\ Shuang LI \\ Xi'an Statistical Research Institute, Xi'an University of Finance \& Economics, Xi'an 710100, China \\ E-mail: lish2006onetwo@163.com
}

\begin{abstract}
Several observations in real economic systems have shown the evidence of non-Gaussianity behavior, and one of mathematical models to describe these behaviors is Poisson noise. In this paper, stationary probability density of a nonlinear business cycle model under Poisson white noise excitation has been studied analytically. By using the stochastic averaged method, the approximate stationary probability density of the averaged generalized FPK equations are obtained analytically. The results show that the economic system occurs jump and bifurcation when there is a Poisson impulse existing in the periodic economic system. Furthermore, the numerical solutions are presented to show the effectiveness of the obtained analytical solutions.
\end{abstract}

Keywords business cycle model; Poisson white noise; stationary probability density

\section{Introduction}

Business cycle modeling is one of the most active subjects in the study of complex economic systems. Recently, a nonlinear business cycle model based on the Samuelson-Hick's consumption function and Puu's investment function has been investigated by researchers ${ }^{[1-3]}$. Puu et al. ${ }^{[1]}$ studied a number of bifurcation sequences leading to attractors and their basins in this model. Li et al. ${ }^{[2]}$ introduced Gaussian white noise excitation into the model and investigated bifurcations under noise excitation. Han et al. ${ }^{[3]}$ considered the reliability of the model with Gaussian white noise excitation by using stochastic averaged method. The above researches show that the nonlinear business cycle model based on the Samuelson-Hick's consumption function and Puu's investment function has complex dynamical phenomena and can be a useful tool to study the real economic behaviors. Researchers in economic modeling have realized that there are many uncertainty factors in a complex economic system and adopted Gaussian white noise as a useful stochastic process to describe several types of economic phenomena ${ }^{[2-7]}$. However, several observations in real economic systems have shown the evidence of non-Gaussianity

Received April 1, 2014, accepted September 2, 2014

Supported by the National Natural Science Foundation of China $(10802061,10972181)$ and Shaanxi Provincial Natural Science Foundation of China (SJ08F27) 
behavior, manifested as in heavy-tail distributions or in impulse of samples ${ }^{[8]}$. Moreover, uncertainty factors in economic systems tend to be discrete events, such as the introduction of new economic and money policies, unexpected political and nature events, all of which cannot be appropriately modeled by Gaussian white noise. In light of these new observations, it is necessary to introduce a new mathematical model to describe these economic systems, and an effective model is the Poisson noise. In this paper, a nonlinear dynamical business cycle model with Poisson white noise excitation is introduced and studied for the first time. The Itó stochastic differential equations and generalized FPK equations from the new model are derived based on the method proposed by Di Paola and Falsone ${ }^{[9]}$. The averaged generalized FPK equation is also derived with correction terms. Finally, the approximate stationary solution of the system is obtained analytically by using stochastic averaged method of single-degree-of-freedom quasilinear systems driven by Poisson white noise ${ }^{[10]}$. The analytical solutions of the new model show the bifurcation and stochastic jumps in business cycles, and numerical results are presented to verify the effectiveness of the analytical solutions.

\section{The Nonlinear Model with Poisson White Noise}

A simple multiplier-accelerator nonlinear dynamical model of business cycle was first induced in [3], which was based on the Hick's consumption function and Puu's investment function together with a cubic nonlinear term under Gaussian white noise,

$$
\ddot{q}+(1-a) \dot{q}+(1+a) \dot{q}^{3}+b q=\beta W(t)
$$

where $q$ is the output. The parameter $a=v-s, a>0$ where $v$ is the 'accelerator', $v>1 ; s$ is the marginal propensity to save, and $0 \leq s \leq 1$. The parameter $b$ is the rate of long-term saving, and $0 \leq b \leq 1$. $W(t)$ is Gaussian white noise. $\varepsilon$ is a small parameter.

Now we introduce three new parameters:

$$
\dot{q}=p, \quad a_{1}^{\prime}=a+1, \quad a_{2}^{\prime}=a-1
$$

and the Poisson noise ${ }^{[10]}$

$$
C(t)=\sum_{i=1}^{N(t)} Y_{i} \delta\left(t-t_{i}\right)
$$

where $\delta$ is a Dirac delta function, $N(t)$ is the Poisson counting process with average impulse $\lambda$, $Y_{i}$ is the impulse intensity, $E\left(Y_{i}\right)=0$ and $E\left[(\mathrm{~d} C(t))^{k}\right]=\lambda E\left[Y^{k}\right] \mathrm{d} t$. By substituting (2) into (1), we obtain the following equations

$$
\begin{aligned}
& \mathrm{d} q=p \mathrm{~d} t \\
& \mathrm{~d} p=-\left(a_{1}^{\prime} p^{3}-a_{2}^{\prime} p\right) \mathrm{d} t-b q \mathrm{~d} t+\varepsilon \mathrm{d} C(t)
\end{aligned}
$$

For simplicity, we let

$$
a_{1}^{\prime}=\varepsilon^{2} a_{1}, \quad a_{2}^{\prime}=\varepsilon^{2} a_{2}
$$

and obtain from (3)

$$
\begin{aligned}
& \mathrm{d} q=p \mathrm{~d} t \\
& \mathrm{~d} p=-\varepsilon^{2}\left(a_{1} p^{3}-a_{2} p\right) \mathrm{d} t-b q \mathrm{~d} t+\varepsilon \mathrm{d} C(t)
\end{aligned}
$$


where $\varepsilon$ is a small parameter. When $\varepsilon=0$, the system is conservative in the absence of the noise term, with Hamiltonian, $H$ of

$$
H=\frac{1}{2} p^{2}+\frac{1}{2} b q^{2}
$$

\section{Averaged Generalized FPK Equations}

To solve the equation, we rewrite (5) in the form of Stratonovich stochastic differential equations:

$$
\begin{aligned}
& \mathrm{d} q=\frac{\partial H}{\partial p} \mathrm{~d} t \\
& \mathrm{~d} H=-\varepsilon^{2}\left(a_{1}\left(\frac{\partial H}{\partial p}\right)^{3}-a_{2} \frac{\partial H}{\partial p}\right) \frac{\partial H}{\partial p} \mathrm{~d} t+\varepsilon \frac{\partial H}{\partial p} \circ \mathrm{d} C(t)
\end{aligned}
$$

According to Itô rule, we introduce the Wong-Zakai correction terms ${ }^{[1,12]}$ and obtain the following Itô stochastic differential equations,

$$
\begin{aligned}
\mathrm{d} q= & \frac{\partial H}{\partial p} \mathrm{~d} t \\
\mathrm{~d} H= & -\varepsilon^{2}\left(a_{1}\left(\frac{\partial H}{\partial p}\right)^{3}-a_{2} \frac{\partial H}{\partial p}\right) \frac{\partial H}{\partial p} \mathrm{~d} t+\varepsilon \frac{\partial H}{\partial p} \mathrm{~d} C(t) \\
& +\frac{\varepsilon^{2}}{2 !} \frac{\partial H}{\partial p} \frac{\partial}{\partial H}\left(\frac{\partial H}{\partial p}\right)[\mathrm{d} C(t)]^{2} \\
& +\frac{\varepsilon^{3}}{3 !} \frac{\partial H}{\partial p} \frac{\partial}{\partial H}\left(\frac{\partial H}{\partial p} \frac{\partial}{\partial H}\left(\frac{\partial H}{\partial p}\right)\right)[\mathrm{d} C(t)]^{3} \\
& +\frac{\varepsilon^{4}}{4 !} \frac{\partial H}{\partial p} \frac{\partial}{\partial H}\left(\frac{\partial H}{\partial p} \frac{\partial}{\partial H}\left(\frac{\partial H}{\partial p} \frac{\partial}{\partial H}\left(\frac{\partial H}{\partial p}\right)\right)\right)[\mathrm{d} C(t)]^{4}+\cdots
\end{aligned}
$$

Using the relation,

$$
\frac{\partial H}{\partial p} \frac{\partial}{\partial H}\left(\frac{\partial H}{\partial p}\right)=\frac{1}{2} \frac{\partial}{\partial H}\left[\left(\frac{\partial H}{\partial p}\right)^{2}\right]=\frac{1}{2} \frac{\partial}{\partial H}\left[2 H-b q^{2}\right]=1
$$

Equation (8) can be simplified to

$$
\begin{aligned}
& \mathrm{d} q=\frac{\partial H}{\partial p} \mathrm{~d} t \\
& \mathrm{~d} H=-\varepsilon^{2}\left(a_{1}\left(\frac{\partial H}{\partial p}\right)^{3}-a_{2} \frac{\partial H}{\partial p}\right) \frac{\partial H}{\partial p} \mathrm{~d} t+\varepsilon \frac{\partial H}{\partial p} \mathrm{~d} C(t)+\frac{\varepsilon^{2}}{2}[\mathrm{~d} C(t)]^{2}
\end{aligned}
$$

Let

$$
g\left(q, \frac{\partial H}{\partial p}\right)=a_{1}\left(\frac{\partial H}{\partial p}\right)^{3}-a_{2}\left(\frac{\partial H}{\partial p}\right)
$$

and by using $E\left[(\mathrm{~d} C(t))^{k}\right]=\lambda E\left[Y^{k}\right] \mathrm{d} t$, the simplified evolution equation of probability density $p(q, h, t)$ associated with Itô SDEs $(10)$ is ${ }^{[10]}$

$$
\begin{aligned}
\frac{\partial p}{\partial t}= & -\frac{\partial}{\partial q}\left(\frac{\partial h}{\partial p} p\right)-\frac{\partial}{\partial h}\left[\left(-\varepsilon^{2} g\left(q, \frac{\partial h}{\partial p}\right) \frac{\partial h}{\partial p}+\frac{\varepsilon^{2}}{2} \lambda E\left[Y^{2}\right]\right) p\right] \\
& +\frac{1}{2 !} \frac{\partial^{2}}{\partial h^{2}}\left[\varepsilon^{2}\left(\frac{\partial h}{\partial p}\right)^{2} \lambda E\left[Y^{2}\right]+\varepsilon^{3} \frac{\partial h}{\partial p} \lambda E\left[Y^{3}\right]+\frac{\varepsilon^{4}}{4 !} \lambda E\left[Y^{4}\right] p\right]
\end{aligned}
$$




$$
-\frac{1}{3 !} \frac{\partial^{3}}{\partial h^{3}}\left[\varepsilon^{3}\left(\frac{\partial h}{\partial p}\right)^{3} \lambda E\left[Y^{3}\right]+\frac{3}{2} \varepsilon^{4}\left(\frac{\partial h}{\partial p}\right)^{2} \lambda E\left[Y^{4}\right] p\right]+O\left(\varepsilon^{4}\right)
$$

According to [13], the conditional probability density $p(q \mid h, t)$ is

$$
p(q \mid h, t)= \begin{cases}\frac{\sqrt{b}}{2 \pi \sqrt{2 h-b q^{2}}}, & b q^{2}<2 h \\ 0, & b q^{2} \geq 2 h\end{cases}
$$

By substituting $p(q, h, t)=p(h, t) p(q \mid h, t)$ into Equation (13) and integrating Equation (13) with respect to $q$, the averaged FPK equation for $p(h, t)$ is

$$
\begin{aligned}
\frac{\partial p}{\partial t}= & -\frac{\partial}{\partial h}\left[\left(-\varepsilon^{2} G(h)-\frac{\varepsilon^{2}}{2} \lambda E\left[Y^{2}\right]\right) p\right] \\
& +\frac{1}{2 !} \frac{\partial^{2}}{\partial h^{2}}\left[\varepsilon^{2} \lambda E\left[Y^{2}\right] h+\frac{\varepsilon^{4}}{4 !} \lambda E\left[Y^{4}\right] p\right] \\
& -\frac{1}{3 !} \frac{\partial^{3}}{\partial h^{3}}\left[\frac{3}{2} h \varepsilon^{4} \lambda E\left[Y^{4}\right] p\right]+O\left(\varepsilon^{4}\right)
\end{aligned}
$$

where

$$
G(h)=\oint \frac{\sqrt{b}}{2 \pi} g\left(q, \frac{\partial h}{\partial p}\right) \mathrm{d} q
$$

Substitute Equation (11) into (15), and let

$$
q=\sqrt{2 h / b} \sin \theta, \quad p=\sqrt{2 h} \cos \theta
$$

One can get

$$
G(h)=\oint \frac{\sqrt{b}}{2 \pi} g\left(q, \frac{\partial h}{\partial p}\right) \mathrm{d} q=h\left(\frac{3}{2} a_{1} h-a_{2}\right)
$$

\section{Approximate Stationary Solution of the Averaged Generalized FPK Equations}

In order to derive the approximate stationary solutions of Equation (14), we rewrite Equation (14) in the following form

$$
\frac{\mathrm{d}}{\mathrm{d} h}\left[\alpha G(h) \rho(h)+\beta h \frac{\mathrm{d} \rho(h)}{\mathrm{d} h}+\frac{1}{4} \gamma h \frac{\mathrm{d}^{2} \rho(h)}{\mathrm{d} h^{2}}+\frac{1}{8} \gamma h^{2} \frac{\mathrm{d}^{3} \rho(h)}{\mathrm{d} h^{3}}+O\left(\varepsilon^{5}\right)\right]=0
$$

where $\alpha=\varepsilon^{2}, \beta=\frac{1}{2} \varepsilon^{2} \lambda E\left[Y^{2}\right], \gamma=\frac{1}{2} \varepsilon^{4} \lambda E\left[Y^{4}\right]$.

From Equation (18), it is easy to know that it can be rewritten as

$$
\alpha G(h) \rho(h)+\beta h \frac{\mathrm{d} \rho(h)}{\mathrm{d} h}+\frac{1}{4} \gamma h \frac{\mathrm{d}^{2} \rho(h)}{\mathrm{d} h^{2}}+\frac{1}{8} \gamma h^{2} \frac{\mathrm{d}^{3} \rho(h)}{\mathrm{d} h^{3}}+O\left(\varepsilon^{5}\right)=0
$$

Equation (19) can be solved by using perturbation technique ${ }^{[14]}$. We obtain the following form of the perturbation solution of Equation (19)

$$
\rho(h)=\rho_{0}(h)+\varepsilon^{2} \rho_{1}(h)+\varepsilon^{4} \rho_{2}(h)+\cdots
$$

Substituting Equation (20) into (19), we get the following equations

$$
\alpha G(h) \rho_{0}(h)+\beta h \frac{\mathrm{d} \rho_{0}(h)}{\mathrm{d} h}=0
$$




$$
\alpha G(h) \rho_{1}(h)+\beta h \frac{\mathrm{d} \rho_{1}(h)}{\mathrm{d} h}=-\frac{1}{4 \varepsilon^{2}}\left(\gamma h \frac{\mathrm{d}^{2} \rho_{0}(h)}{\mathrm{d} h^{2}}+\frac{1}{2} \gamma h^{2} \frac{\mathrm{d}^{3} \rho_{0}(h)}{\mathrm{d} h^{3}}\right)
$$

..

To solve Equation (21) subject to the non-negative condition and normalization condition, one can get

$$
\begin{aligned}
& \rho_{0}(h)=c_{1} \exp \left(-\frac{\alpha h\left(3 a_{1} h-4 a_{2}\right)}{4 \beta}\right) \\
& \rho_{1}(h)=c_{1} \exp \left(-\frac{\alpha h\left(3 a_{1} h-4 a_{2}\right)}{4 \beta}\right) \frac{\alpha \gamma}{64 \beta^{4} \varepsilon^{2}} m(h)
\end{aligned}
$$

where

$$
\begin{aligned}
m(h)= & \frac{27}{5} \alpha^{2} a_{1}^{3} h^{5}-\frac{27}{2} \alpha^{2} a_{1}^{2} a_{2} h^{4}+\left(12 \alpha^{2} a_{1} a_{2}^{2}-30 \alpha \beta a_{1}^{2}\right) h^{3} \\
& +\left(42 \alpha \beta a_{1} a_{2}-4 \alpha^{2} a_{2}^{3}\right) h^{2}+\left(24 a_{1} \beta^{2}-16 \alpha \beta a_{2}^{2}\right) h
\end{aligned}
$$

Substituting (22) and (23) into (20), we get the approximate stationary solution up to order of $\varepsilon^{4}$ as following

$$
\rho(h)=\rho_{0}(h)+\varepsilon^{2} \rho_{1}(h)=D \exp \left(-\frac{\alpha h\left(3 a_{1} h-4 a_{2}\right)}{4 \beta}\right)\left(1+\frac{\alpha \gamma}{64 \beta^{4}} m(h)\right)
$$

where

$$
D=\left[\int_{0}^{\infty} \exp \left(-\frac{\alpha h\left(3 a_{1} h-4 a_{2}\right)}{4 \beta}\right)\left(1+\frac{\alpha \gamma}{64 \beta^{4}} m(h)\right) \mathrm{d} h\right]^{-1}
$$

Now we assume that the intensity of random pulse, $Y$ conform Gaussian distribution with zero mean for illustrative purpose. So $E\left[Y^{4}\right]=3\left(E\left[Y^{2}\right]\right)^{2[10]}$. Meanwhile, the stationary probability density for system state $q$ and $p$ can be calculated as follows

$$
\rho(p, q)=\rho(q, h)\left|\frac{\partial(q, h)}{\partial(p, q)}\right|=\rho(h) \rho(q \mid h) p=\left.\frac{\sqrt{b}}{2 \pi} \rho(h)\right|_{h=\frac{1}{2} p^{2}+\frac{1}{2} b q^{2}}
$$

\section{Dynamics of the Model}

In order to get the approximate stationary probability density and the numerical solution of energy $H$ for the business cycle model, we fix the parameters as $\varepsilon=0.5, \lambda=2, b=0.5$. By using Monte Carlo method, we get Figures 1-6 as follows.

Figures 1 and 2 with $a_{1}=0.2, a_{2}=1.0$, and Figures 3 and 4 with $a_{1}=1.0, a_{2}=1.0$. All the figures show that the analytical solutions correspond to the numerical solutions very well.

In Figure 1, it's seen that the system is stable as the probability density $\rho(h)$ reaches the max value when the total energy of the economic system reaches a certain value. Moreover, from Figure 2, we know that the 'double peaks' of the marginal probability density of displacement $q$ would take place in the situation when there exist impulse in the periodic economic system, in which the intensities of the impulse are (a) $<Y^{2}>=0.1$; (b) $<Y^{2}>=1.0$, respectively. With the intensity of the impulse decreasing from $\left\langle Y^{2}\right\rangle=7.0$ to $\left\langle Y^{2}\right\rangle=0.1$, the marginal probability density of displacement $q$ evolutes from 'single peak' to 'double peaks'. Namely, a bifurcation occurs under this circumstance. In other words, the economic system becomes unstable by jumping between different stable situations, which will result in economic crisis in this periodic economic system. 


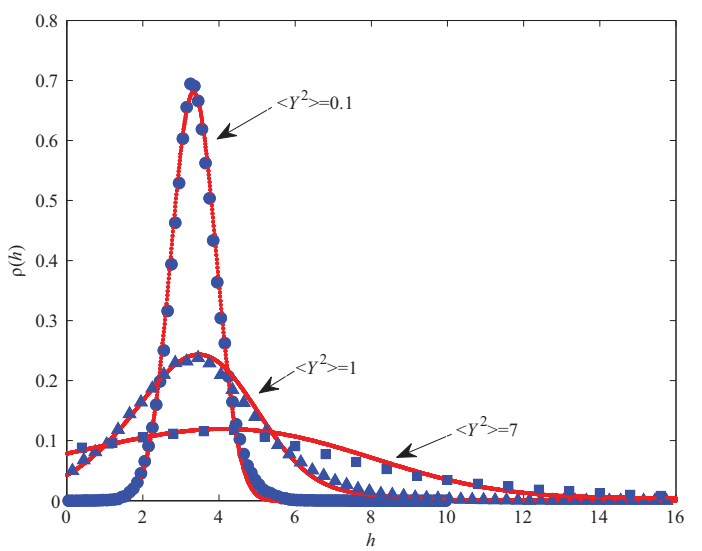

Figure 1 The stationary probability density of energy $H$ for the business cycle model with $a_{1}=0.2, a_{2}=1.0$; (a) $<Y^{2}>=0.1$, (b) $<Y^{2}>=1.0$, (c) $<Y^{2}>=7.0$. - the approximate stationary solution; $\bullet \mathbf{\square} \boldsymbol{\Delta}$ the simulation results

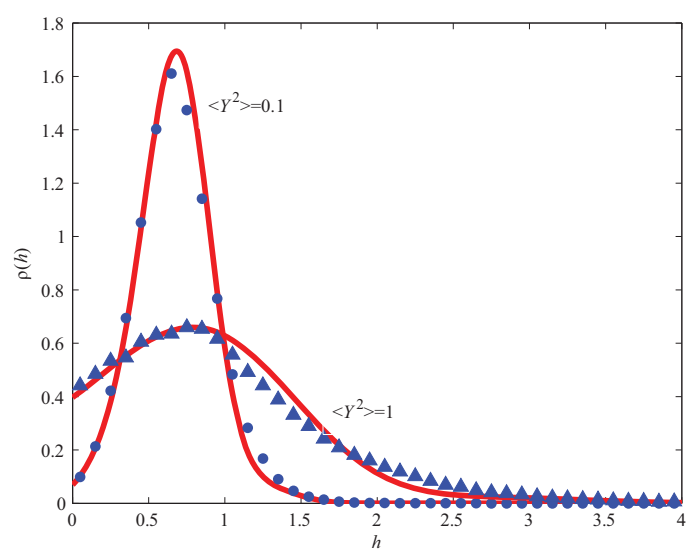

Figure 3 The stationary probability density of energy $H$ for the business cycle model with $a_{1}=1.0, a_{2}=1.0$; (a) $<Y^{2}>=0.1$, (b) $<Y^{2}>=1.0$. - the approximate stationary solution; $\bullet \boldsymbol{\square}$ the simulation results

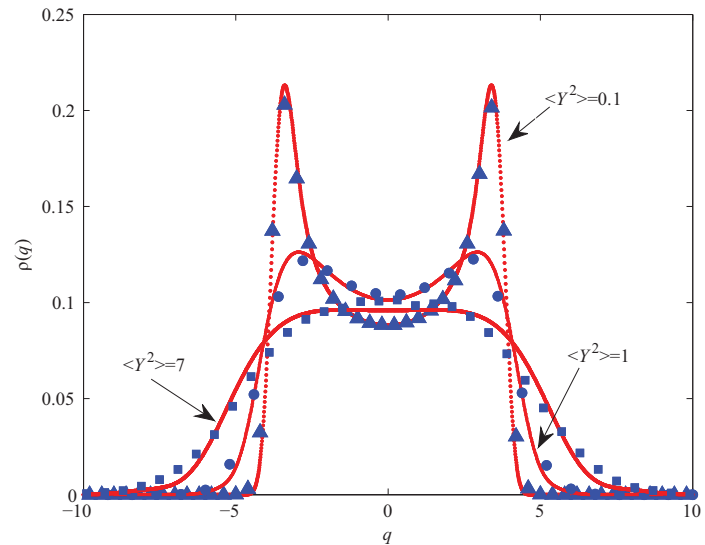

Figure 2 The stationary marginal probability density of displacement $q$ for the business cycle model with $a_{1}=$ $0.2, a_{2}=1.0$; (a) $<Y^{2}>=0.1$, (b) $<Y^{2}>=1.0$, (c) $<Y^{2}>=7.0$. - the approximate stationary solution; $\bullet \mathbf{\Delta}$ the simulation results

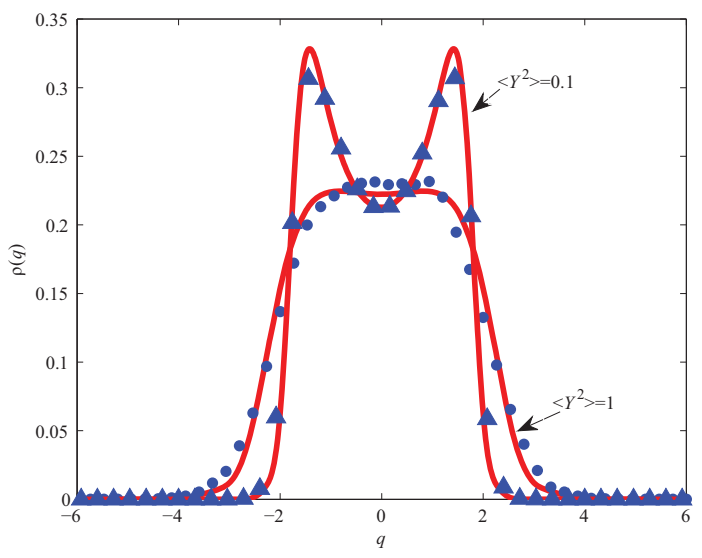

Figure 4 The stationary marginal probability density of displacement $q$ for the business cycle model with $a_{1}=$ $1.0, a_{2}=1.0 ;$ (a) $<Y^{2}>=0.1$, (b) $<Y^{2}>=1.0 . \quad-$ the approximate stationary solution; $\bullet \mathbf{\square}$ the simulation results 
From Figures 3 and 4 , with the values of the parameters $a_{1}, a_{2}$ changing, namely the 'accelerator' and the marginal propensity, the system responses are similar to Figures 1 and 2. This phenomenon further verifies that the marginal probability density of displacement $q$ of the system response evolves from 'single peak' to 'double peaks', a nd bifurcation takes place in the process.

In Figures 5 and 6 , when $a_{1}=1.5$ and $\left\langle Y^{2}\right\rangle=1$, by adjusting the value of $a_{2}$, the equilibrium point of the system will change, but not the topologic shape of the marginal probability density of the response.

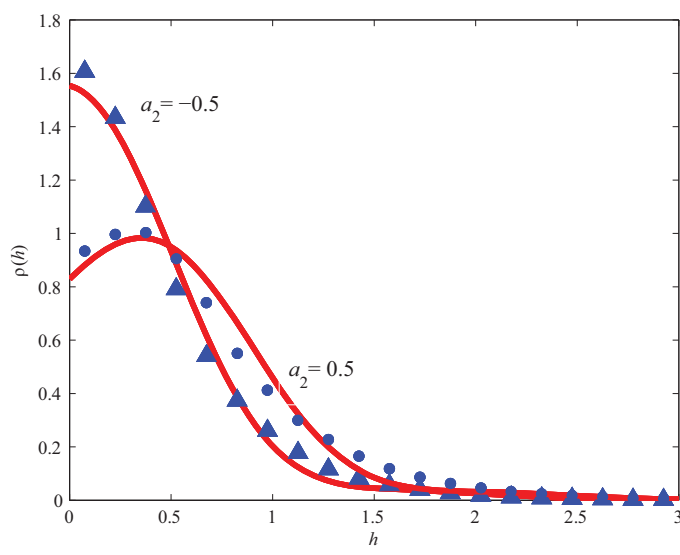

Figure 5 The stationary probability density of energy $H$ for the business cycle model with $a_{1}=1.5,<Y^{2}>=1$; (a) $a_{2}=-0.5$, (b) $a_{2}=0.5$. - the approximate stationary solution;

$\boldsymbol{\Delta} \boldsymbol{\Delta}$ the simulation results

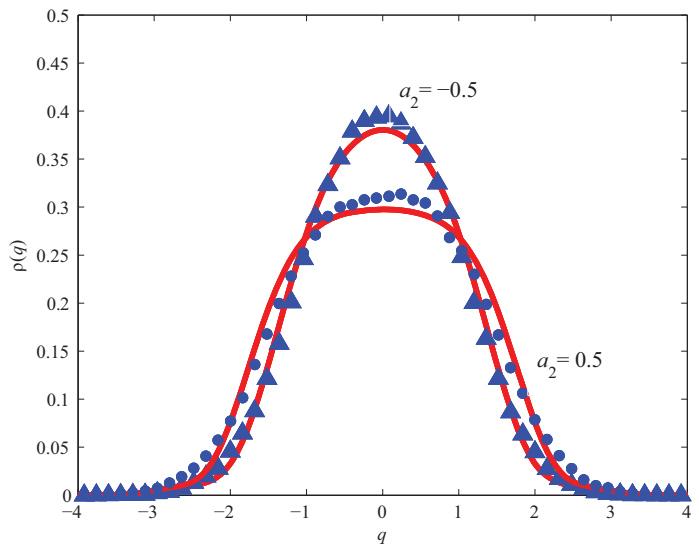

Figure 6 The stationary marginal probability density of displacement $q$ for the business cycle model with $a_{1}=$ $1.5,<Y^{2}>=1$; (a) $a_{2}=-0.5$, (b) $a_{2}=0.5$. - the approximate stationary solution; $\bullet \boldsymbol{\square}$ the simulation results

Comparing the present results with Shen's ${ }^{[2]}$, we find that the business cycle system doesn't bring about Hopf bifurcations in $H=0$ when Poisson white noise is introduced to the equation. In reality, the total energy will never reach zero, and the system will be stable just in non-trivial total energy. In brief, Poisson white noise is better than Gaussian white noise to describe the uncertainty in economical system.

\section{Conclusions}

In this paper, we investigated the stationary probability density of a nonlinear model under Poisson white noise excitation. Based on the averaged Itô stochastic differential equations and generalized FPK equations of the stochastic model, the approximate stationary probability density of the averaged generalized FPK equations were obtained by using the perturbation method. For some given parameters, the numerical solutions went well with the analytical solutions. From the results we found that the economic system would have incurred jumps and bifurcations when there exists a Poisson impulse in the system itself. 


\section{References}

[1] Puu T, Sushko I. A business cycle model with cubic nonlinearity. Chaos, Solitons \& Fractals, 2004, 19: 597-612.

[2] Li W, Xu W, Zhao J F. Stochastic stability and bifurcation in a macroeconomic model. Chaos, Solitons \& Fractals, 2007, 31(3): 702-711

[3] Han H, Wang H, Xu J, et al. Reliability analysis based on stochastic model of business cycle. Advanced Management of Information for Globalized Enterprises, 2008, 9: 28-29.

[4] Li W, Zhao J. Bifurcation and chaos in a macroeconomic model. International Journal of Nonlinear Sciences and Numerical Simulation, 2006, 7: 431-434.

[5] Li W, Xu W, Xu Y, et al. First-passage and its control in a macroeconomic model. Far East Journal of Applied Mathematics, 2007, 28: 1-16.

[6] Li J, Ren Z, Wang Z. Response of nonlinear random business cycle model with time delay state feedback. Physica A, 2008, 387: 5844-5851.

[7] Li J, Li S. Dynamics of a business cycle model under harmonic and stochastic noise excitation. Far East Journal of Applied Mathematics, 2010, 38: 119-129.

[8] Cont R, Tankov P. Financial modeling with jump processes. New York: Chapman \& Hall/CRC, 2004, 7-13.

[9] Di Paola M, Falsone G. Itô and Stratonovich integrals for delta-correlated processes. Probabilistic Engineering Mechanics, 1993, 8: 197-208.

[10] Zeng Y, Zhu W Q. Stochastic averaging of quasi-linear systems driven by Poisson white noise. Probabilistic Engineering Mechanics, 2010, 25: 99-107.

[11] Di Paola M, Falsone G. Stochastic dynamics of non-linear systems driven by non-normal delta-correlated processes. Journal of Applied Mechanics, 1993, 60: 141-148.

[12] Di Paola M, Santoro R. Path integral solution for non-linear system enforced by Poisson white noise. Probabilistic Engineering Mechanics, 2008, 23: 164-169.

[13] Stratonovich R L. Topics in the theory of random noise. Vol. 1, New York: Gordon and Breach, 1963.

[14] Cai G Q, Lin Y K. Response distribution of nonlinear-systems excited by non-Gaussian impulsive noise. Internaltional Journal of Non-Linear Mechanics, 1992, 27: 955-967. 